\title{
The meaning of death
}

\author{
Gerry Melino*,1 \\ 1 IDI-IRCCS, c/o University Tor Vergata, 00133 Rome, Italy. \\ * Corresponding author: G Melino; E-mail: gerry.melino@uniroma2.it \\ DOI: $10.1038 / \mathrm{sj} / \mathrm{cdd} / 4401001$
}

Albert Camus, following Novalis and Kierkegard, puts suicide at the centre of thinking. Since over $2 \%$ of all 650000 papers published annually in the life sciences are related to apoptosis, it would seem that biology has also entered an existentialist phase. ${ }^{1,2}$

It is the balance between cell choices (mitosis, differentiation and apoptosis) which determines that the overall number of cells within an adult organism and within each of its individual organs remains essentially constant. However, while mitosis and differentiation have received detailed attention from cell and molecular biologists for well over a century, a morbid fascination with cell death has only recently become a major interest. Still, it is incredible that for a long time biologists never questioned the fate of so many duplicating cells in our body.

Part of the explanation for this historical imbalance of interest lies in the apoptotic process itself. Apoptosis is executed roughly 20 times as rapidly as mitosis, and the apoptotic cell is also rapidly absorbed and degraded by neighbouring cells, this very speed making scientific observation and description more difficult. Indeed, $20 \%$ of mitotic cells are apparently balanced by $1 \%$ of apoptotic cells, the limit of detection. Moreover, apoptosis does not leave any residual tell-tale signs after it has occurred. The apoptotic cell effectively becomes surrounded by an impermeable cocoon which prevents extracellular leakage of any potentially harmful intracellular contents, and which would otherwise cause surrounding inflammation and scarring. This also serves to distinguish apoptosis from the passive, toxic cell death (necrosis) where extracellular leakage and inflammation are distinctive features. The introduction of the concept of apoptosis and its molecular interpretation, which is still only partially understood, ${ }^{3}$ now allows us to understand how these cellular choices are made.

Thomas Kuhn, like Gunther Stent, argues that a discovery occurs with the natural perception of an anomaly, when a series of implications could not be linked by the current logic - a process which can take a long time. Cell death clearly shows such a long gestation. In this issue of Cell Death and Differentiation David Vaux has prepared a very nice timeline of cell death publication chronology. ${ }^{4}$ Even though it is difficult to cite everybody in a field where so many pivotal contributions have been made, Vaux elegantly succeeds in giving a schematic graphical representation. Some morphological observations which we would now regard as apoptotic have been made since the middle of the 19th century, though their biological significance has been appreciated only recently. In 1842, Vogt recognised a form of physiological cell death, while Flemming, in 1855, used the term 'chromatolysis' to describe the nuclear fragmentation seen during cell death - a characteristic still used, among others, as a hallmark of apoptosis. Other similar descriptions occurred occasionally in the XIXth and early XXth centuries. More recently, the embryologist Gluckmann (1951), the haematologist Bessis (1955), and the biologist Tata (1960) clearly described the morphological phases of apoptosis. In the 1960s, working on insect development, Lockshin recognised the coordinated death of sheets of cells - a process he termed 'Programmed Cell Death' - and which he showed to be energy dependent and to require gene transcription. In 1966 John Saunders was able to review 'Death in embryonary systems', showing the role of cell death in moulding the body during development. A little later, Kerr, Wyllie and Currie described a similar cell death morphology in mammals, but this time affecting individual cells within a tissue, and for which they used the term Apoptosis.

It was not until apoptosis moved from the morphological to the mechanistic that it fully acquired scientific credibility and began to provide an intellectual framework for the previously scattered observations. ${ }^{3}$ For this, the credit largely goes to Robert $\mathrm{H}$ Horvitz and his collaborators (Michael Hengartner and Junying Yuan). ${ }^{5,6}$ Working with the nematode, Caernorhabditis elegans, Horvitz first mapped the fate of every cell in the organism, including those that were to commit apoptosis. By 1985 he had shown that apoptosis was determined by several genes (The Terminators), including ced-9 (The Good, which blocks apoptosis), ced-3 (The Bad, which executes apoptosis) and ced-4 (The Ugly, which is an activator of apoptosis). This work created a new intellectual framework, moving from observation to interpretation, and thus played a pivotal role in the creation and diffusion of the concept. This has been highly conducive to scientific development, as evident from the number of genes and pathways identified and their emerging physiological and pathological roles. The 3 main genes have been highly conserved throughout evolution, such that they, or rather their corresponding gene families, still determine the apoptotic process in mammals. Thus, in man, there are over 21 Goods (the Bcl-2 family), 14 Bads (the caspase family), but still (so far) only one Ugly (Apaf-1). Caspases can be the target of viral (as first shown by Lois Miller) or cellular (BIR) proteins modulating cell death, and are now being actively exploited for pharmaceutical purposes, for example by Don Nicholson's work. ${ }^{3,7}$ The molecular effector mechanisms were clarified with the brilliant work of Xiaodong Wang and the definition of a novel dedicated cellular organelle, the 
apoptosome, which activates the Bads. Like all biological systems, however, death (like life) is not as simple as that, and apoptosis following mitochondrial damage or death receptor binding may not be inevitable. A number of regulatory sites have been described which can interfere with proapoptotic signalling, and even some caspase-like molecules have been shown to have anti-apoptotic effects. The Bads are not always so bad? This multiplicity of interacting pro- and anti-apoptotic factors implies that, as in mitosis, a number of checks and balances are present in the apoptotic programme. In the present issue Eugene Koonin has prepared a very detailed and comprehensive analysis of the evolutionary pattern of the proteins and the relative structural domains involved in cell death. ${ }^{8}$ From this, the original links with bacterial proteins, often involved in distinct functions, and the rise in complexity incurred during the evolution of the species is evident. A more specific description of only one class of proteins crucial for cell death, the caspases, is presented by Peter Vandenabeele. $^{9}$

It is therefore only within the closing years of the last millennium, The Golden Age, that apoptosis has been given the scientific interest that its biological significance deserves, providing important research guidelines for the next decade. ${ }^{10,11}$ More recently, differences in the execution of apoptosis are emerging. Possibly, these new emerging facets of the original concept are related to cellto-cell variation due to the fact that several molecular constituents of the process exist as large redundant molecular families which are differently expressed in different tissues. ${ }^{3}$

The biology of apoptosis has now reached the level of a fashion. With over 13,000 papers annually and two dedicated journals, it may be that overemphasis is leading to distortion. The concentration on apoptotic death diminishes the physiological and pathological role of necrosis and fields such as toxicology are desperately searching for a new identity. At the same time, the definition is changing, and acquiring subtle differences. Originally, the term 'programmed cell death' was a morphological definition while 'apoptosis' implied a biochemical character (apoptosis=caspases). Now, the two terms are frequently used as synonymous, as opposites to necrosis (passive, non genetically programmed death). The term 'cell death' has become more general, including not just apoptosis and necrosis but also other types of death such as: autophagy, caspase-independent-cell death, keratinisation and erythrocyte karyorexis. Until definitions are linked to biochemical pathways, however, these subdivisions remain semantic.

In 1992 Martin Raff developed the concept of 'social control of life and death'. ${ }^{12}$ This concept is of enormous power, implying specific survival and death molecular signals and their corresponding receptors. In fact, independently from the $C$. elegans model, Peter Krammer and Shigekazu Nagata identified the first death receptor, CD95, and its ligand CD95L in the early 1990s. ${ }^{3}$ Receptors, and their related signals, are of particular importance in multicellular systems, such as the immune system, where signalling is crucial. This implies that the concept of cell death developed late in evolution. Raff's model of apoptosis is linked to multicellularity, and its development therefore follows evolutionary complexity (e.g. immune and neural systems).

In 1995 Jean-Claude Ameisen found that cell death can be applied also to unicellular organisms. ${ }^{13}$ In fact, JC Ameisen, P Golstein and JC Reed have described forms of cell death in bacteria, Tetrahymena and Dictyostelium. Possibly a major event in this is the chimeric-symbiotic origin of the eukaryotic cell when a Dangerous Liaison was established with the mitochondria. Now Ameisen revisits all these concepts in an excellent detailed review. ${ }^{14}$ In his review, Jean-Claude Ameisen provides examples of repressor models, and in particular toxin-antidote modules, in evolution. This follows, at a more scientific level, his highly recommended book. ${ }^{1}$ In this frame of thinking, and following the links between infection and immunity, Doug Green puts forward the concept that apoptosis is related to infection. ${ }^{15}$ Indeed, infections, immunity, and apoptosis seems to be very tightly related.

It is suggested that on the occasion of the centennial of the Nobel Prize, Apoptosis was chosen as the main topic. ${ }^{16}$ This emphasizes the importance reached by this field.

I have to personally thank Jean-Claude Ameisen for the several exciting conversations on The meaning of life over dinner, drinking Bourgogne, that stimulated these articles. Similarly, Doug Green, David Vaux, Dick Knight and I indulged in innumerable evening thoughts over glasses of Chianti, Sheraz and Napa Valley. I only hope that the reader enjoys the articles as much as we enjoyed the wine!

\footnotetext{
1. Ameisen JC (1999) La sculpture du vivant. Ed. du Seuil, Paris

2. Melino G (2001) Nature 412: 23

3. Nature Insight on Apoptosis (2000) Nature 407: 769-816

4. Vaux DL (2002) Cell Death Differ. 9: 349-354

5. Horvitz HR et al (1982) Neuroscience Commentaries 1: 56-65

6. Horvitz HR (1999) Cancer Res. (Suppl.) 59: 1701s-1706s

7. Nicholson DW (1999) Cell Death Differ. 6: 1028-1242

8. Koonin EV and Aravind L (2002) Cell Death Differ. 9: 394-404

9. Lamkanfi M et al (2002) Cell Death Differ. 9: 358-361

10. Garfield E and Melino G (1997) Cell Death Differ. 4: 352-361

11. Melino $\mathrm{G}$ et al (2001) Cell Death Differ. 8: $1-3$

12. Raff MC (1992) Nature 356: $397-400$

13. Ameisen JC et al (1995) Cell Death Differ. 2: 285-300

14. Ameisen JC (2002) Cell Death Differ. 9: 367-393

15. James ER and Green DR (2002) Cell Death Differ. 9: 355-357

16. Robertson JD et al (2002) Cell Death Differ. 9: 468-475
} 